\title{
Research on Structural Stress Optical Fiber Testing Technology Based on Brillouin Scattering
}

\author{
Xian-Jin WANGa ${ }^{a}$ Qing $L I^{b}$ and Shuai WAN \\ College of Mechanical and Electrical Engineering, China Jiliang University,HangZhou, ZheJiang, China \\ awangxianjin028@126.com, b lq13306532957@163.com
}

\begin{abstract}
In this paper, the principle of distributed optical fiber measurement and measurement of axial stress is introduced by analyzing the principle of Brillouin scattering in an optical fiber with a certain power. Making the experimental device, measuring the individual strain model, analyzing the wave shape of the scattered wave, and preliminary understanding of the image characteristics of the stress in the optical fiber Brillouin scattering spectrum. The effect of stress on the different position of the fiber, and the difference between them and the scattering waveform obtained from the stress free action poetry are compared, and the effect of the light pulse on the optical fiber transmission is studied. The results show that the effect of the stress is different in the position of the action, and the effect of the propagation of the pulse light is mainly affected by the Stokes and anti Stokes light scattering. The research can provide reference for distributed optical fiber measurement, and it can promote the application of distributed optical fiber in measuring micro deformation. The innovative point of this study is to use the pulley method to solve the effect of the different position of the same stress in the distribution of optical fiber.
\end{abstract}

\section{Guidelines}

The propagation of optical fiber in the optical fiber, because the fiber is a non crystalline material, the uneven structure in the micro space, so there is a small amount of scattering phenomenon. Brillouin scattering is the scattering of light waves propagating in the optical fiber, which is generated by the interaction between the light wave and the sound wave.

Large hoisting equipment is very common in industrial equipment, with China's rapid urbanization, large heavy equipment in industrial production everywhere, heavy lifting equipments take heavy beams by stress and strain is not intuitive performance, so frequent over expression of construction will be to the naked eye is difficult to observe the damage created by force, local regions of relatively large deformation, causing potential safety hazard. These deformation can be observed through the strain of the small changes in the micro stress, so as to achieve the purpose of prevention. The distributed observation of the deformation stress can be realized by the Brillouin scattering effect of the optical fiber in the optical fiber.

\section{Distributed Optical Fiber Sensing Technology Based on Brillouin Scattering}

\subsection{Distributed Optical Fiber Sensing Technology Based on Brillouin Optical Time Domain Reflection Analyzer}

BOTDA technique is a kind of optical time domain analysis technique based on stimulated Brillouin scattering. Implantation certain frequency $f$ of the optical pulse signal at one end of the single-mode fiber. At the other end of the injection frequency $f$ is the center of the fluctuation $f_{B}$ continuous probe, in the two beams of light to meet the position changes of stimulated Brillouin scattering, $f+f_{B}$ frequency continuous probe in this position will provide gain or attenuation, thus all the places in the entire length of the fiber optical power transformation can be detected in the time domain. So as to realize the measurement of environmental variables of distributed optical fiber. The basic principle is shown in Figure 1: 


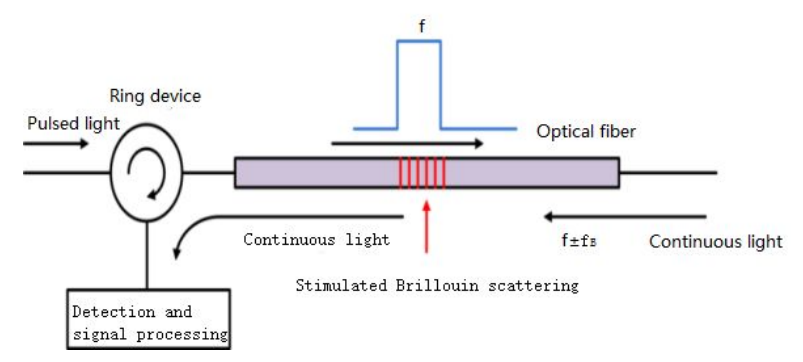

Figure 1. The technology principle of Brillouin optical time-domain analysis

\subsection{The Relationship between the Frequency of Brillouin Scattering and Axial Strain}

According to the optical signal of Brillouin frequency shift and linear relationship between the optical fiber temperature and axial strain, fiber optic strain variation in temperature compensation (or strain compensation) conditions (or temperature) by the formula (1) said:

\section{$\Delta \mathrm{V}_{\mathrm{B}}=\mathrm{Cvt} \Delta \mathrm{t}+\mathrm{Cv} \varepsilon \Delta \varepsilon(1)$}

Type, $\Delta V_{B}$ as the Brillouin frequency shift, the Cvt for Brillouin frequency shift temperature coefficient, $\mathrm{Cv} \varepsilon$ as a Brillouin frequency shift to strain coefficient, $\Delta \mathrm{t}$ is the change of temperature, $\Delta$ sis the change of strain variation. In this experiment we ignore the effect of temperature on the Brillouin frequency shift (assuming constant temperature), which is that temperature does not change or change range is very small, so the Brillouin frequency shift and stress there is a clear linear relationship.

\section{Design and Construction of Experimental Platform}

\subsection{Design and Fabrication of Experimental Device}

The experimental device is designed as a pipeline structure, which is distributed to a single mode optical fiber, and makes the stress distribution model. The frequency shift pattern of the Brillouin scattering wave is measured by the BOTDA equipment. Experimental pipeline section size is $0.6 \mathrm{~m}$ external diameter is $0.7 \mathrm{~m}$, the length is $7 \mathrm{~m}$, at the bottom of the conjoined tripod overall to the in the distance from the center of the total length of $6 \mathrm{~m}$ to prop up, and in the center of the device to add pipeline strain is applied on the part and support part can be fixed screw, when the strain at the middle, both ends of the free will not move. On one side of the pipe to take way of grinding to make it a smooth surface, easy laying optical fiber, after the completion of the layout, in order to make the strain effect reaches the maximum, here we put fiber on the applied strain device on the side, namely the whole fiber layout part in the cross section of the pipeline from the ground at the lowest point. In the pipeline, the distance between each part of the tag is put up to facilitate the analysis of the frequency shift between the distance and the Brillouin scattering. After the completion of the production of the overall device as shown in figure 2 .

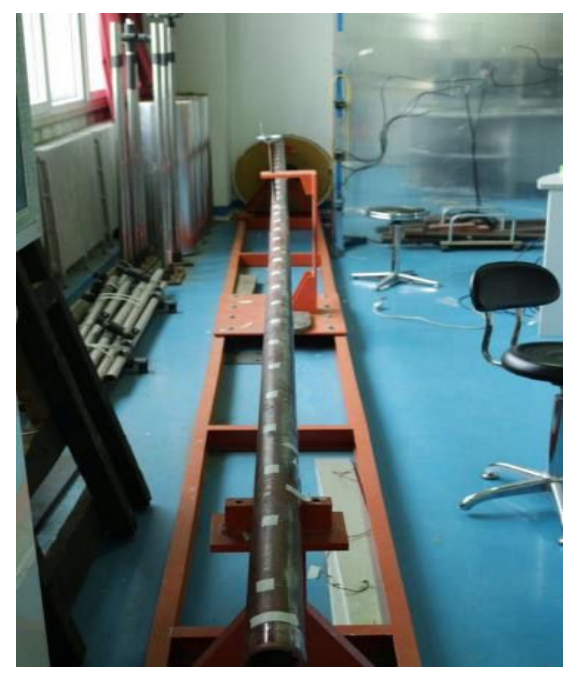

Figure 2. Integral device complete graph

\subsection{Experimental Instruments and Related Parameters Setting}

The instrument used in this experiment is a distributed optical fiber temperature and strain monitoring system DiTeST STA-R. The instrument is in the XP Windows system under the data acquisition and analysis instrument, after opening the instrument, in the parameter adjustment zone as shown in the following table 1 Parameters of the setting, after the completion of the setting can be started.

Table 1. Ditest Sta-R Device Parameter Setting

\begin{tabular}{|l|l|}
\hline Instrument parameters & Set value \\
\hline spatial resolution & $0.5(\mathrm{~m})$ \\
\hline Sampling distance & $0.1(\mathrm{~m})$ \\
\hline Start frequency & automatic detection \\
\hline Sensing optical fiber length & automatic detection \\
\hline
\end{tabular}

\subsection{Study on the influence of Brillouin scattering frequency shift}

\subsubsection{Effect of Different Axial Stresses on Frequency Shift}

Because the bare fiber is easy to break, so in this experiment we take is with two movable pulley can move the optical fiber fixing way, as shown in Figure 3, 1, 4 lines coincide, and in the graph at the left end of the fixed 1 , line 4 , the right end of the dynamic fixed pulley, and the left end of the movable pulley moves to the right position, observe the Brillouin frequency shift and the free section (fixed in the pipeline not stress fiber), getting the frequency change as shown: 


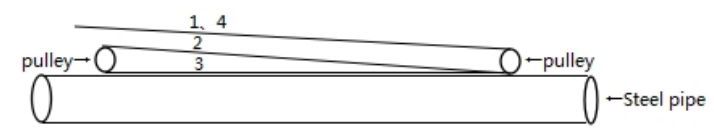

Figure 3. Bonding arrangement of fiber on steel pipe

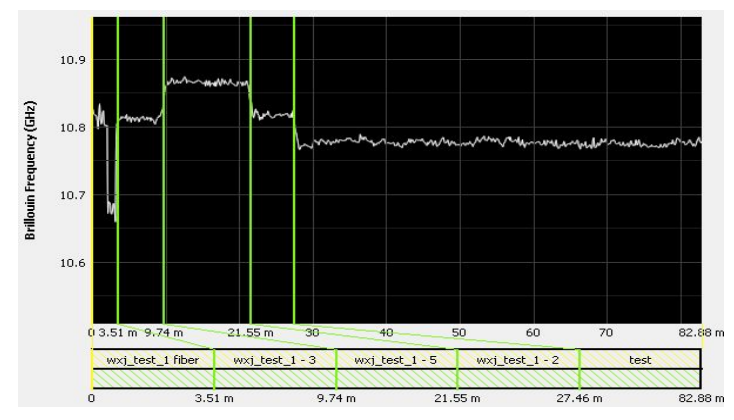

Figure 4. Map distance distribution of Brillouin frequency space

We can see from Figure $4(27.46-82.88 \mathrm{~m})$ of the free end of the Brillouin frequency is $10.78 \mathrm{GHz}$, relative to the free section can be seen in the section we applied axial stress (3.51-27.46m) have different degrees of freedom with respect to the frequency of the mobile terminal, wherein the $0-3.51 \mathrm{~m}$ is pulse light emission opening to start jumper cable length between fixed ends of BOTDA instrument $3.51-9.74 \mathrm{~m}$, No. 1 fiber frequency fixed, $9.74-21.55 \mathrm{~m}$ is 2,3 fiber frequency state left movable sheave connected, 21.55-27.46 4 fiber frequency fixed state, we can see from Figure 2, 3, 1, 4, relative to the optical fiber optical fiber has a significantly different frequency mobile, this is because we have 1,2 of the left and right pulley is fixed, when we move the left wheel, right around the fiber due to friction pulley existence, guide To the 1, 4 and 2, 3 optical fiber has significantly different frequency shift, in order to verify the conclusion, from the new design of a test pulley friction between the $1,4,2,3$, and the frequency difference between the fiber.

\subsubsection{The Effect of Friction Force on the Experimental Results of Pulley}

In the last experiment based, continue to shift left the pulley on the left, and the No. 2 fiber toward the direction of fiber 1 slightly manual marathon relaxed a little and let the free recovery, fiber optic frequency as shown in Figure 5 below:

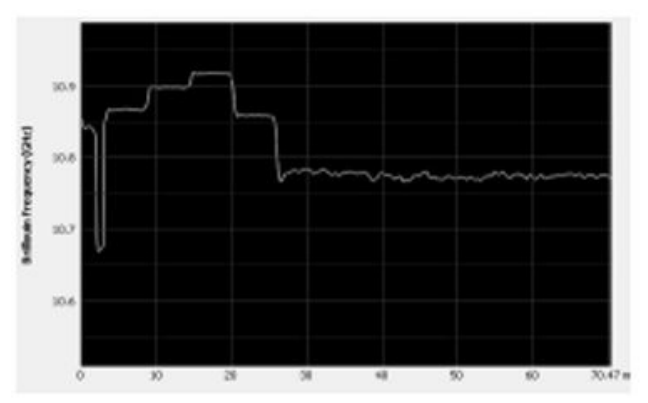

Figure 5. Effect of friction between the pulley of Brillouin frequency shift
As shown in the figure, the total length of the optical fiber to 70.47. This is because in the experimental process, fiber breaking a, from the new layout of the fiber, the length of the measurement of the fracture part for $12.2 \mathrm{~m}$, combined with optical fiber fusion intercepted length is about $0.2 \mathrm{~m}$ and so reduce length is 12.4 , combined with $82.88-70.47=12.41 \mathrm{~m}$. Therefore on Brillouin scattering measurement of fiber length accuracy is very high. Analysis diagram of intermediate frequency shift size we can see 3 optical frequency shift relative to the $1,2,4$, theory on 1, 2, 3, 4 optical frequency shift should is the same, but due to the existence of the friction between the pulley around, resulting in stress on 1, 2, 3, 4 fiber on is force varies is the result of the above results.

\subsubsection{Frequency Shift Effect of Different Stresses}

Thereafter from the newly laid optical fiber, and constantly increase the stress at the center, the application of stress devices such as the following figure 6 :

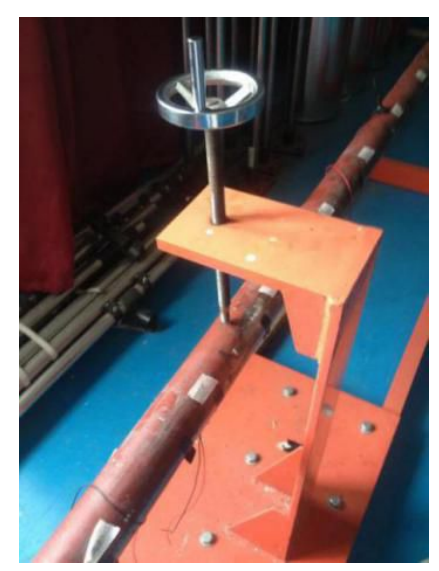

Figure 6. The middle of the pipeline booster

The different stress frequency shift images are obtained as shown in figure 7 :

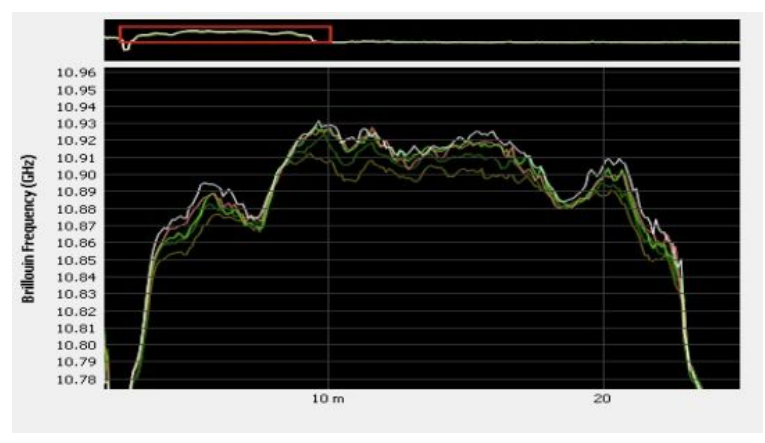

Figure 7. Different Brillouin frequency state corresponding to the stress gradient

Figure from brown to white frequency curve are never should be the initial state of the force to the maximum stress of 5 should be gradient force corresponding to the frequency, can be roughly seen with the increase of stress, the frequency corresponding move and by the graph we can see and 1, 4 optical fiber is directly connected on the right side of the pulley is basically no frequency shift of 
(figure around $8.5 \mathrm{~m}$ and $18.5 \mathrm{~m}$ or so), but the pulley on the left junction has a frequency shift $(12 \mathrm{~m})$, where speculation is different on both sides of the pulley friction, leading to the pulley on the left than the right side pulley frequency shift occurred.

The maximum frequency shift and non stress time frequency shift can be obtained frequency shift effect chart, as follows

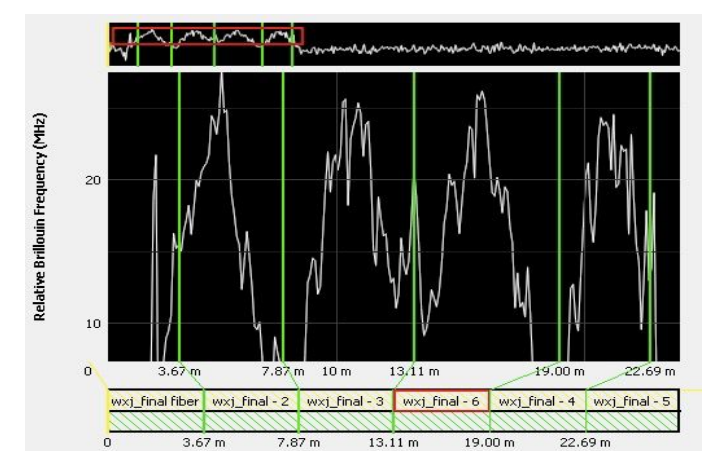

Figure 8. Comparison between different stress gradients

From the figure we can seen in our design of pulley fiber this experiment, 1, 2, 3, 4 fiber in the center position of the tube have taken place in the frequency shift, and in both sides of the maximum stress decreasing, size and frequency shift is basically the same, can draw Brillouin frequency shift depends on the initial state of the state of optical fiber has nothing to do, and by force is proportional to the and the maximum should force near presenting reduced state. The average length of fiber bonding is(22.69-3.67) $/ 4=4.755 \mathrm{~m}$, combined with the frequency shift of the 4 sections of the state, you can see that the frequency of each section of the gradient of nearly 5 gradient, so that its spatial resolution is $4.755 / 5$, nearly $1 \mathrm{~m}$.

\section{Conclusion}

Through the experiment to verify the distributed optical fiber measurement of axial stress of Brillouin scattering frequency shift effect, provides the theoretical support for the distributed optical fiber Chang'an Technology in engineering application, and take this as the basis for quantitative analysis of the frequency shift and should be the relationship between the force, and explore the distributed optical fiber sensing technology of maximum spatial resolution and sensing accuracy.Compared with the traditional optical fiber layout and pulley arrangement can intuitively find Brillouin frequency shift is obviously position, so as to achieve the best experimental results.

\section{References}

1. Zhang Chun shu, Bao Xiao yi, Ozkan Istemi F, et al. Prediction of thepipe buckling by using broadening factor with distributed Brillouin fiber sensors[J]. Optical Fiber Technology, 2008, 14(2): 109-113

2. Yi Liqi, Qian Caifu,Ding Keqin.A method for calculating deformation of long-distance pipleline based on strain. Advanced Materials Reasearch.(EI)2011,vols.291-294:3385-3390

3. Yi Liqi,Qian Caifu,Ding Keqin,Qiao Song.A method for detecting deformation of pipleline based on distributed fiber optic strain sensor. Advanced Materials Reasearch. (EI) 2011, vols. 301303:1574-1577

4. Bao Xiao-yi, MERCHANT M De, BROWN A,et.al. Tensile and compressive strain measurement in the lab and field with the distributed Brillouin scattering sensor[J].Journal of Lightwave Technology, 2001, 19(11):1698-1704

5. Zhang C, Bao X, Ozkan I F, et al. Prediction of the pipe buckling by using broadening factor with distributed Brillouin fiber sensors[J]. Optical Fiber Technology, 2008, 14(2): 109-113 